\title{
Desarrollo de habilidades para la vida en Escuela Móvil: estrategia de empoderamiento de niños y adolescentes como sujetos de derecho
}

\author{
Development of skills for life in mobile school: strategy for the empowerment of children and \\ adolescents as subjects of right \\ María Lizzeth Chaves-Oviedo'* orcid.org/0000-0003-3962-4788 \\ Álvaro Darío Dorado-Martínez' orcid.org/0000-0002-9879-5109
}

1 Universidad de Nariño. Pasto, Colombia

Fecha de recepción: Julio 4 - 2018

Fecha de revisión: Diciembre 7 - 2018

Fecha de aceptación: Agosto 26 - 2019

Chaves-Oviedo ML, Dorado-Martínez AD. Desarrollo de habilidades para la vida en Escuela Móvil: estrategia de empoderamiento de niños y adolescentes como sujetos de derecho. Univ. Salud. 2019;21(3):205-214. DOI: http://dx.doi.org/10.22267/rus.192103.157

\section{Resumen}

Introducción: Las habilidades para la vida son un conjunto de características que permiten al individuo actuar de manera adecuada en la cotidianidad, facilitando un buen crecimiento físico, emocional e intelectual. Objetivo: Empoderar a los niños y adolescentes como sujetos de derecho desde el fortalecimiento de habilidades para la vida. Materiales y métodos: Paradigma cualitativo, con una metodología de investigación acción en donde se desarrolló la estrategia de Escuela Móvil con un enfoque basado en derechos humanos, a través de una serie de talleres realizados con 20 niños y adolescentes entre los 6 y 14 años. Resultados: Los niños y jóvenes adquirieron y fortalecieron un repertorio conductual en defensa de su infancia y se vincularon a actividades comunitarias. Conclusiones: Escuela Móvil logró el empoderamiento de los niños y adolescentes como sujetos de derechos, quienes reconocieron claramente sus derechos fundamentales, los deberes por los cuales deben responder y además participaron de manera activa y constante de la realidad comunitaria.

Palabras clave: Adolescentes; derechos del niño; toma de decisiones. (Fuente: DeCS, Bireme).

\begin{abstract}
Introduction: Life skills are a set of characteristics that allow individuals to act properly in a daily manner, facilitating an appropriate physical, emotional and intellectual development. Objective: To empower children and adolescents as subjects of rights, through the strengthening of life skills. Materials and methods: A qualitative paradigm with an action-research approach that developed a mobile school strategy which included a series of workshops with 20 children and adolescents whose ages ranged between 6 and 14 years. Results: Children and young people acquired and strengthened a behavioral repertoire in defense of their childhood and also engaged in community activities. Conclusions: Mobile School empowered children and adolescents as subjects of rights, who clearly recognized their fundamental rights and duties and also actively and constantly participated in the community reality.
\end{abstract}

Key words: Adolescent; rights of the child; decision making. (Source: DeCS, Bireme).

\footnotetext{
*Autor de correspondencia

María Lizzeth Chaves Oviedo

e-mail: marialic0717@gmail.com
} 


\section{Introducción}

Ecuador es un país con una población cercana a los 15 millones de habitantes, está constituido por 24 provincias, entre ellas Manabí con 22 cantones, en donde se encuentra el cantón Bolívar, su cabecera cantonal es Calceta y tiene dos parroquias rurales, Membrillo y Quiroga(1). En los últimos años la Parroquia Quiroga ha registrado un bajo nivel de escolaridad, déficit de servicios básicos en la mayoría sus comunidades, aumento de desnutrición crónica en niños menores de cinco años (45\%) y mayor incidencia de violencia intrafamiliar, consumo de alcohol, tráfico y uso de drogas lícitas e ilícitas; problemáticas que se originan desde los núcleos familiares en donde no se evidencia un rol claro entre padres e hijos(2), generando que los niños y jóvenes no contemplen una figura de autoridad y hayan adquirido ciertos comportamientos caracterizados por la rebeldía y el incumplimiento de la norma.

Reconociendo que los programas de educación en habilidades para la vida ayudan a niños y jóvenes a desarrollar estas características por medio de métodos de enseñanza interactivos que incluyen actuaciones, discusiones abiertas, ensayo de habilidades y actividades en grupos pequeños, se creó "Escuela Móvil”, como una estrategia hecha para niños y adolescentes, con un enfoque basado en derechos humanos. Para el desarrollo de esta, se tuvo en cuenta la educación popular la cual basa la adquisición del aprendizaje en las experiencias y el contexto social; es decir, exige la generación de prácticas y saberes que permitan el análisis crítico de la realidad, la acción colectiva y el desarrollo de valores sociales democráticos(3).

Las personas como seres sociales por naturaleza establecen relaciones con aquellos que los rodean en los distintos entornos: familiar, educativo, laboral $\mathrm{u}$ ocio(4). Para la creación de Escuela Móvil se tuvo en cuenta la definición y clasificación de las habilidades para la vida de la Organización Mundial de la Salud (OMS) que toma al autoconocimiento, empatía, comunicación asertiva, relaciones interpersonales, toma de decisiones, solución de problemas y conflictos, pensamiento creativo, pensamiento crítico, manejo de emociones, sentimientos y manejo de tensiones y estrés, como las diez habilidades importantes en la promoción de la salud que permiten mejorar la capacidad para vivir sanamente y feliz, interviniendo sobre los determinantes de la salud, el bienestar y participando de manera activa en la construcción de sociedades justas, solidarias y equitativas $(5)$.

Las habilidades para la vida, por lo tanto, son un conjunto de características que permiten al individuo actuar de manera competente y habilidosa en las distintas situaciones de la cotidianidad y el entorno, favoreciendo así comportamientos saludables, aportando en la promoción de la salud, la prevención de problemas y la comunicación con los demás; lo cual facilita un adecuado crecimiento físico, emocional e intelectual y un desarrollo personal, familiar y social pleno(6).

Dentro del ámbito de la salud se ha demostrado que enseñar a los niños y adolescentes a desarrollar las habilidades para la vida es la forma más eficaz para establecer comportamientos saludables tanto de manera individual como colectiva. La evaluación de programas que incluyen el desarrollo de estas habilidades, muestra que su fortalecimiento puede retrasar e inhibir el uso de drogas, prevenir conductas sexuales de alto riesgo, enseñar a controlar la ira, mejorar el desempeño académico y promover el ajuste social positivo(7).

El proceso de Escuela Móvil, desde su enfoque basado en derechos humanos, planteó respetar a la infancia $y$ adolescencia en cuanto a sus singularidades, su proceso de autonomía, reconociendo su participación como actores activos de cambio en los espacios sociales con los que interactúan, siendo sujetos capaces de promover y proteger sus derechos y los de otros, a la vez que como ciudadanos se comprometen con lo público y el bien común y actúan con relación a valores como autonomía, libertad y responsabilidad frente a las situaciones que comprometen su dignidad y la de los demás(8).

Reconocer a los niños y adolescentes como sujetos de derecho significa que les corresponden los mismos derechos, deberes y garantías que a los adultos y otros derechos especiales(9), entre los cuales se encuentran el derecho la vida, educación, alimentación, agua, salud, identidad, libertad y protección, estos y cada uno de los derechos son inalienables e irrenunciables, por lo tanto ninguna persona puede vulnerarlos o desconocerlos en ninguna circunstancia(10). 
El Estado y la ciudadanía adulta en su conjunto son los responsables de garantizar y procurar la máxima satisfacción de tales derechos, en Ecuador el Código de la Niñez y Adolescencia establece las acciones que regulan las normas encaminadas a la protección de los derechos infantiles y su principal desafío es superar la desigualdad y lograr un desarrollo pleno en cada una de las etapas del crecimiento(11).

De esta manera la investigación se realizó con una muestra de la población infantil y adolescente de la Parroquia Quiroga y tuvo como objetivo empoderar a los niños y adolescentes como sujetos de derecho desde el fortalecimiento de habilidades para la vida, basándose en el paradigma cualitativo, con una metodología de investigación acción en donde se desarrolló la estrategia de Escuela Móvil con un enfoque basado en derechos humanos.

\section{Materiales y métodos}

Esta investigación fue de tipo cualitativo con un enfoque crítico social y una metodología de investigación acción que permite la expansión del conocimiento y el planteamiento de respuestas concretas a problemáticas comunes, de tal manera que los participantes se convierten en coinvestigadores del proceso(12-14).

\section{Población y muestra}

La Parroquia Quiroga tiene 3.767 habitantes, de los cuales 1.516 son niños y adolescentes entre los $0 \mathrm{y}$ 19 años. Se trabajó con una muestra de Veinte menores de edad, 9 hombres y 11 mujeres cuyas edades oscilaban entre los 6 y 14 años; habitantes de los barrios Miramar, Bajo grande, La pavita, y La pita de la Parroquia Quiroga.

Se utilizó el Muestreo no probabilístico por conveniencia, teniendo en cuenta que la participación se dio de forma voluntaria a partir del interés y motivación de los niños frente al proceso, de esta manera llegaron cinco representantes de cada barrio a conformar Escuela Móvil. Además, se contó con la participación de los padres de familia, docentes, adultos que conformaban el GAD Parroquial (Gobierno Autónomo Descentralizado) y la comunidad en general, quienes fueron los agentes facilitadores de las actividades y los constantes evaluadores del proceso.

\section{Criterios de inclusión}

Niños y niñas cuyas edades oscilaban entre los 6 y 14 años y residieran de los barrios Miramar, Bajo grande, La pavita, y La pita, de la Parroquia Quiroga. Estos barrios fueron escogidos debido a las distintas problemáticas que se presentaban de forma recurrente, relacionadas con la convivencia, violencia intrafamiliar, consumo de sustancias psicoactivas, deserción escolar y condiciones económicas precarias.

Contar con autorización de los padres para que los niños participaran de las actividades propuestas en el proceso y disposición de tiempo y motivación de los niños para hacer parte de Escuela Móvil.

\section{Instrumentos y técnicas de recolección de información}

\section{Observación participante}

Durante el proceso se utilizó el diario de campo como método interactivo de recolección de información, para registrar aquellos hechos que son susceptibles de ser interpretados, siendo una herramienta que permitió sistematizar las experiencias para luego analizarlas ${ }^{(15,16) .}$

\section{Talleres participativos}

Los conocimientos se adquirieron a través de una práctica concreta, realizando actividades relacionadas con aquello que se desea enseñar utilizando esta metodología en la que se enseña y se aprende a través de una tarea conjunta(17).

\section{Procedimiento}

La Escuela Móvil se desarrolló entre abril y noviembre del año 2017 con las siguientes fases:

\section{Contextualización}

La inserción a la comunidad de Quiroga partió del reconocimiento del territorio y de las personas que viven en este lugar, por medio de la observación participante y entrevistas informales con actores sociales como el presidente del GAD Parroquial, docentes y representantes de barrios.

\section{Planificación}

Escuela Móvil contó con cuatro ejes, tres de los cuales abordaron un tema general, dos habilidades para la vida y dos derechos; el otro eje presentaba un tema general, cuatro habilidades para la vida y dos 
derechos. Cada uno de los ejes se desarrolló a través de encuentros de dos semanas; cada uno proponía un taller, siendo el resultado del proceso de identificación de aquellas necesidades que la población infantil de la Parroquia Quiroga presentaba.

\section{Acción}

Los encuentros se realizaron dos veces por semana durante ocho meses, cada sesión se estructuró de acuerdo con el eje correspondiente incluyendo una dinámica inicial, la actividad central y finalmente el espacio de evaluación y retroalimentación.

\section{Evaluación}

Se realizó cada dos meses a partir de una actividad propuesta por los niños, en donde se vinculó a toda la comunidad.

\section{Reflexión}

Se realizó una jornada de socialización del proceso con la comunidad de Quiroga, en esta, los niños narraron la experiencia y realizaron una jornada artística en donde dieron conocer sus habilidades y talentos.

\section{Consideraciones éticas}

La participación de los niños y adolescentes en Escuela Móvil fue voluntaria, se hizo la presentación de la propuesta de investigación a en el GAD Parroquial, al finalizar el encuentro los interesados firmaron junto con sus padres los consentimientos y asentimientos informados. Para fines de esta investigación se tuvo en cuenta la Ley 1090, por la cual se reglamenta el ejercicio de la profesión de Psicología, se dicta el Código Deontológico y Bioético. Con base en ello, la investigación se acogió a las siguientes disposiciones estipuladas en la ley: responsabilidad, confidencialidad, estándares morales y legales, y bienestar del usuario(18). Asimismo, se contó con el aval institucional de la Universidad de Nariño y la Fundación Centro de Orientación comunitaria Infantil Manabí.

\section{Resultados}

\section{Contextualización}

El acercamiento con los principales agentes puso en evidencia las distintas necesidades de la población, refirieron preocupación por el aumento de deserción escolar, casos de violencia intrafamiliar, inicio de consumo de sustancias psicoactivas en edades tempranas, mal manejo del tiempo libre, falta de sentido de pertenencia por la comunidad y dificultades en la comunicación y en el establecimiento de relaciones interpersonales

El foco de atención se centró en la niñez y adolescencia, convocando a una reunión comunitaria en donde se expresó el interés por iniciar un trabajo con esta población, de esta manera, quienes estuvieron interesados firmaron los respectivos consentimientos $\mathrm{y}$ asentimientos informados $\mathrm{y}$ fueron citados a un próximo encuentro.

En el primer encuentro realizado con niños y jóvenes se realizó un taller participativo que tuvo como objetivo el develamiento de las necesidades y problemáticas que ellos evidenciaban en la comunidad y las cuales generaban consecuencias en su desarrollo, como resultado de esta actividad se encontró que no existía un conocimiento de los derechos, razón por la cual en muchas ocasiones se veían vulnerados y por otra parte la necesidad de adquirir y fortalecer las habilidades para la vida, debido a las distintas problemáticas relacionadas con una inadecuada relación entre los sujetos y los distintos entornos con los cuales interactuaban.

\section{Planificación}

Teniendo en cuenta el diagnóstico inicial se planteó Escuela Móvil con dos temas centrales: los derechos humanos y las habilidades para la vida, con el fin de empoderar a los niños y adolescentes como sujetos de derecho. Este proceso se convirtió en un espacio de constante crecimiento, donde los niños y adolescentes desde su experiencia diaria desarrollaban y fortalecían sus habilidades para la vida, reconociendo así sus derechos, se vinculaban a las actividades cotidianas de la comunidad, por medio de espacios propuestos por ellos mismos, logrando que las personas los reconocieran como miembros partícipes importantes en la toma de decisiones frente a situaciones sencillas de su entono (Tabla 1).

\section{Eje 1: Estoy Bien}

Se propuso la actividad física como forma de reconocimiento y adquisición de hábitos saludables que mejorarían la calidad de vida. Se realizaron actividades que incluían ejercicios de resistencia, fuerza $y$ flexibilidad. Tras los conocimientos adquiridos, los niños atribuyeron al ejercicio diferentes beneficios y lo incluyeron en su día a día, 
se reunían en las tardes para jugar futbol, baloncesto, salir en bicicleta o caminar por las calles de Quiroga, esto se convirtió en una forma de interactuar y compartir con los demás.

Tabla 1. Ejes para el fortalecimiento de las habilidades para la vida y reconocimiento de los derechos de la infancia

\begin{tabular}{|c|c|c|c|}
\hline Ejes & Tema & Habilidades & Derechos \\
\hline Estoy bien & Actividad física & $\begin{array}{l}\text { Manejo de emociones } \\
\text { Manejo de tensiones }\end{array}$ & $\begin{array}{l}\text { Derecho a la alimentación } \\
\text { Derecho a la salud }\end{array}$ \\
\hline Seamos creativos & Territorio y comunidad & $\begin{array}{l}\text { Pensamiento crítico } \\
\text { Pensamiento creativo }\end{array}$ & $\begin{array}{l}\text { Derecho al agua } \\
\text { Derecho a la identidad }\end{array}$ \\
\hline Caminar juntos es caminar con otros & Lazos de amistad & $\begin{array}{l}\text { Autoconocimiento } \\
\text { Comunicación asertiva } \\
\text { Empatía } \\
\text { Relaciones interpersonales }\end{array}$ & $\begin{array}{l}\text { Derecho a la educación } \\
\text { Derecho a la protección }\end{array}$ \\
\hline Como soy, como actuó & Derechos de la infancia & $\begin{array}{l}\text { Solución de problemas y conflictos } \\
\text { Toma de decisiones }\end{array}$ & $\begin{array}{l}\text { Derecho a la vida } \\
\text { Derecho a la libertad }\end{array}$ \\
\hline
\end{tabular}

Con relación a la actividad física se abordaron los derechos a la salud y alimentación develando las dificultades que tiene la comunidad para acceder a una atención eficiente y oportuna en el centro de salud; los niños referían "cuando yo me enfermo, no voy al médico porque siempre está cerrado el hospital, entonces mi mamá me prepara aguas aromáticas y con eso ya me siento mejor", también se pudo conocer que sólo ocho de los veinte niños cumplían con el esquema de vacunación completo y tenían al menos una vez al año control médico. Con esta información los niños incentivaron a sus padres para que solicitaran jornadas médicas en la Parroquia las cuales fueron programadas dos veces al año con el fin de velar por el bienestar y la salud de niños, jóvenes, adultos y ancianos.

En cuanto a la alimentación, los niños en Escuela Móvil dieron a conocer cuáles eran los alimentos que consumían en el colegio, en sus hogares y en los lugares que frecuentaban y se encontró que las golosinas, gaseosas, helados y frituras eran más frecuentes que las frutas, verduras y alimentos hechos en casa "A mí me gusta comprar en la hora del descanso algo de picar, en mi escuela venden papas fritas, dulces, jugos y con mis amigos compramos todo eso para compartirnos", "A mí no me gustan las verduras, las frutas si y en mi casa tenemos un árbol de mango y otro de mandarina, mi papá me dice que lleve eso para el descanso pero yo prefiero lo que venden allá". Tras los encuentros los niños expresaron comprender la importancia de incluir en su dieta diaria alimentos saludables que les aportan en su desarrollo físico y mental, llevando diariamente al colegio porciones de fruta y una botella con agua. Conjuntamente con los padres de familia se realizaron dos jornadas en la institución educativa sobre alimentación saludable, las cuales serán replicadas dos veces en el mes con el fin de que los integrantes de la comunidad adopten estos hábitos.

Por otra parte, se abordaron temas relacionados con la forma de comunicación y expresión de sentimientos y emociones; los niños acogieron con gran interés las explicaciones de aquellas técnicas que les permitían manejarlos adecuadamente, fue así como mediante las actividades lograron identificar cuales hacen parte de su diario vivir y cómo emergen frente a situaciones cotidianas. De igual manera. se simularon espacios en donde ellos tenían la oportunidad de exteriorizar su sentir y comunicarlo a los demás de una manera asertiva. Los niños y adolescentes demostraron un reconocimiento de aquellas situaciones que originan tensiones $y$ conflictos e identificaron formas de manejarlos a través de puestas en escena. Todo esto contribuyó a mejorar el ambiente en los distintos contextos de interacción de los niños, permitiendo el diálogo con pares y adultos.

\section{Eje 2: Seamos Creativos}

Este espacio promovió el pensamiento crítico y creativo desde el reconocimiento de la comunidad, el territorio, los derechos al agua y la identidad. Se invitó a los niños y jóvenes a reflexionar frente al significado que la comunidad tiene para ellos, destacando la importancia de las tradiciones y costumbres que han acogido, aquellas necesidades que se presentan, las características que los identifican y la relevancia de ser parte en las actividades comunitarias que se desarrollan cada 
año "A mí me gusta vivir en Quiroga porque es muy bonito y tranquilo, hay lugares para jugar y tengo muchos amigos" "cada año en junio organizamos todo lo que vamos a hacer porque Quiroga también cumpleaños y lo celebramos juntos".

Se trabajó el sentido de comunidad a partir de encuentros con adultos mayores de la Parroquia, quienes a través de historias contaban anécdotas, cambios y todo el crecimiento de Quiroga desde su origen hasta la actualidad. El reconocimiento de la identidad de los niños y adolescentes como "Quirogenses" y la importancia de esta como derecho, permitió que se involucraran en las celebraciones parroquiales, cantonales, provinciales y nacionales, haciendo distintas muestras de canto, música, bailes y representaciones en donde destacaron sus habilidades artísticas y creativas "es bueno que para las actividades que realizan y nos tengan en cuenta porque antes me aburría todo era para los grandes, ahora también pudimos cantar y bailar... me gustó mucho cuando la gente nos aplaudía y felicitaba".

También se tuvo en cuenta el territorio como un espacio con características y límites geográficos que compone el lugar que habitan, los niños y jóvenes asumieron distintos compromisos frente a la protección de este, creando las mingas comunitarias que tenían como fin la unión de las personas para mejorar el aspecto físico de cada barrio, limpiando y organizando los distintos sectores "el sábado todos mis vecinos salieron a la minga, unos barrían, limpiaban y pintaban... después el barrio se miraba muy bonito, todos lo decían". Los niños y adolescentes de Escuela Móvil cumplieron un papel fundamental a la hora de convocar a la comunidad siendo muy dinámicos y propositivos, además estas jornadas permitieron el fortalecimiento de lazos relacionales en la comunidad, siendo un espacio para compartir desde el diálogo y reconocimiento del otro como vecino.

Entre los temas que tuvieron mayor acogida fueron la necesidad de tener acceso al agua potable y la socialización del plan de gestión de riesgo, frente al primero los niños hicieron varias jornadas en la institución educativa con el fin de sensibilizar a la comunidad frente al derecho que tienen todos al agua "Yo no sabía que el agua era un derecho y cuando empezamos a hablar de eso en la escuela, escuche que varios compañeros no tenían agua limpia en sus casas" "después de lo que se hizo en el colegio mi papá junto a otros están haciendo reuniones para que las casas tengan agua limpia y podamos tomarla".

Para el plan de gestión de riesgo se llevaron a cabo charlas con voluntarios de la Cruz Roja Ecuatoriana, quienes brindaron información sobre la forma adecuada en que los niños y jóvenes deben actuar ante emergencias naturales "antes cada vez que había terremoto me asustaba mucho y empezaba a llorar, cuando vinieron los señores de la cruz roja nos explicaron dónde ir, que hacer y que debemos estar tranquilos", los niños participaron con una marcha en la conmemoración del 16 de abril, al ser un año del terremoto en Manabí que causó grandes daños a nivel estructural, pérdidas humanas y afectaciones psicológicas; desde Escuela Móvil, se trabajaron escenarios con el fin de realizar un duelo frente a esta situación, fortaleciendo la resiliencia.

\section{Eje 3: Caminar juntos es caminar con otros}

Se identificó la importancia del reconocimiento individual y el fortalecimiento de los lazos de amistad, desarrollando habilidades de comunicación asertiva y adecuadas relaciones interpersonales. Los encuentros de Escuela Móvil al propiciar la interacción entre sujetos de un mismo grupo etario permitieron identificar la importancia de una comunicación constante y respetuosa, de esta manera, ellos mismos crearon normas de convivencia que garantizaron la ejecución de actividades y la integración de todos los participantes "cuando iniciamos Escuela Móvil nadie estaba juicioso, todos jugaban, gritaban y se peleaban, pero después entendimos que por ser niños no debíamos ser molestosos y que debíamos respetarnos".

La interacción entre los niños proporcionó el autoconocimiento de sus fortalezas y aspectos a mejorar. El solo hecho de que la comunidad fuera partícipe directa de la evolución y logros que tenía el proceso, hacía que la autoestima de los niños aumentara de manera notable, de tal manera que cada uno valoraba sus pensamientos, acciones y sentimientos "antes me daba miedo participar en el parque porque pensaba que no podía o que iba a salir mal, me gusta que ahora nuestros papás esperen nuestra presentación y pregunten que vamos a hacer".

Los lazos de amistad entre los niños y jóvenes se reforzaron de manera constante a través de toda la investigación, evidenciando empatía y comunicación 
asertiva a la hora de reconocerse diferentes y por ende reconocer la realidad del otro, los niños se mostraban solidarios y capaces de aportar en los momentos difíciles que atravesaban las personas "yo no hablaba casi con nadie, pero en Escuela Móvil ya tengo muchos amigos y compartimos todos los días, estamos pendientes de todos hasta de los más pequeños" "yo he aprendido a no molestar a mis amigos, a escucharlos y brindarles mi amistad".

Asimismo, se identificó que en los hogares no existía un rol claro de los padres y una representación de autoridad de los mismos, por esta razón fueron vinculados a algunos de los encuentros con el fin de brindarles la oportunidad de expresar sus gustos y compartir distintas dinámicas para comprender el papel que tienen como padres, a fin de garantizar a los niños un ambiente que enriquezca su conocimiento, personalidad. Todos los niños que hicieron parte de Escuela Móvil contaban con su mamá y papá o uno de los dos estaba a cargo, existen algunas dificultades económicas debido a las pocas oportunidades laborales de la región, sin embargo, en cuanto a la educación todos los niños tienen acceso a la escuela y el colegio de la Parroquia, garantizando su formación hasta el bachillerato.

\section{Eje 4: Como soy, como actuó}

En este eje se abordaron las habilidades relacionadas con solución de problemas, conflictos y toma de decisiones y los derechos a la vida y la libertad. Los encuentros se basaron en la presentación de casos de la vida cotidiana, puestas en escena y situaciones que incluyeran la realidad social del país con el fin de que los niños y adolescentes dieran a conocer su postura, frente a estos acontecimientos y tuviesen la oportunidad de plantear acciones y tomar decisiones frente a las mismas "acá en Escuela Móvil aprendí que también puedo dar mi opinión y que todo lo que sucede nos puede afectar".

En relación con el derecho a la vida se abordó la violencia intrafamiliar y el consumo de sustancias psicoactivas, dos hechos que durante los últimos años han aumentado su incidencia en la Parroquia, inicialmente los niños no presentaron mayor interés frente a estos temas y preferían evadir o no participar en las mesas de discusión que se proponían "en mi casa no hablamos de drogas porque mi mamá siempre se queda callada, pero hay muchos conocidos en el colegio que las usan y es bueno que alguien grande nos explique qué pasa con eso y en
Escuela Móvil aprendí todo el daño que pueden causar". Sin embargo, tras develar que son ellos la población más vulnerable empezaron a involucrarse, primero reconociendo las rutas a seguir $y$ posteriormente, llevando a la comunidad cine foros, obras de teatro, fotografías desde las cuales se generó concientización frente al tema.

Por su parte, el derecho a la libertad se trabajó desde la importancia de respetar la opinión y expresión del otro, los niños aprendieron a tomar la palabra, escuchar al otro y dar su punto de vista de manera respetuosa, el trato entre ellos demostraba el proceso realizado siendo muy importante la comprensión, tolerancia y ver a los demás como seres humanos con sentimientos y emociones "en el colegio nos han felicitado porque ya no nos burlamos cuando alguien se equivoca es que era muy gracioso cuando leían y en el descanso les poníamos apodos yo no me daba cuenta que eso los ponía tristes y ya no lo hacemos".

Se considera que este eje fue transversal por cuanto su evaluación fue constante en cada una de las actividades que incentivaban a los niños a buscar una solución y tomar una decisión que beneficiara a todos. Principalmente se movilizó entorno a la defensa de los derechos de la infancia, en donde los pequeños ya tenían un diálogo y accionar que promovía su respeto "nuestros derechos son muy importantes y como somos pequeños los adultos deben cuidarnos, pero también podemos decir cuando algo no está bien" "Escuela Móvil me dio amigos, conocimientos... los niños también somos Quiroga".

\section{Evaluación}

Después de cada encuentro los participantes de Escuela Móvil recibían una hoja de retroalimentación de las actividades propuestas y tenían un espacio para dar a conocer cómo se sentían con el proceso, cuáles eran sus aprendizajes y sugerencias; de esta manera, se podía realizar los cambios requeridos con el fin de alcanzar el objetivo propuesto.

El proceso de adquisición y fortalecimiento de habilidades para la vida y el reconocimiento de los derechos humanos fue evaluado por medio de una actividad comunitaria cada dos meses, de esta manera se vinculó a padres, docentes y comunidad en general recibiendo sus recomendaciones e indagando cuáles eran los cambios que observaban, gracias a esto Escuela Móvil se estructuraba 
conforme a la evolución que se evidenciaba en los participantes.

\section{Reflexión}

Se hizo un análisis de los resultados obtenidos por medio de los diarios de campo, hojas de evaluación de los encuentros y actividades comunitarias. Al tener un informe consolidado se realizó un encuentro con la comunidad para socializar el proceso realizado; los niños y adolescentes que participaron en la investigación cumplieron un papel clave en este espacio, relatando su experiencia y haciendo una puesta en escena de sus habilidades artísticas y creativas. De esta manera, se pudo constatar la importancia de trabajar las habilidades para la vida y los derechos humanos para que los niños y adolescentes puedan reconocerse como sujetos de derecho capaces de actuar y decidir frente a las distintas situaciones que se presentan en su Parroquia.

\section{Discusión}

La investigación cualitativa plantea la importancia de identificar la naturaleza de las realidades, la estructura dinámica y las relaciones que se establecen, dando razón del comportamiento y manifestaciones con el fin de recoger datos, categorizarlos e interpretarlos; así el conocimiento es el resultado de una dialéctica entre el investigador y el objeto de estudio (19). A través del proceso investigativo se pudo constatar la importancia de conocer y ser parte del contexto por cuanto desde la cotidianidad de las acciones realizadas por los actores se pudo establecer la base para crear estrategias y procesos que contribuyeran a la organización y fortalecimiento de procesos comunitarios como lo plantea Melero(13), quien destaca la importancia de estar comprometido no sólo con la explicación de la realidad sino con la transformación de la misma, a través de una dinámica liberadora y emancipadora de las personas que de un modo u otro se encuentran implicadas.

Dado que Escuela Móvil fue evaluada constantemente los participantes tuvieron la posibilidad de reflexionar sobre sus acciones y replantearlas según los objetivos propuestos, asumiendo su rol como sujetos de derechos, capaces de defenderlos, respetarlos y, asimismo, cumplir con sus deberes. Lo anterior coincide con la definición que le da Kurt Lewin a la investigación acción, siendo una experiencia reflexiva social en la que interactúan la teoría y la práctica con miras a establecer cambios apropiados en la situación estudiada y en la que no hay distinción entre lo que se investiga, quién investiga y el proceso de investigación(20).

Teniendo en cuenta que las habilidades para la vida han despertado el interés en su estudio, se retoma a World Vision Costa Rica, una organización sin fines de lucro que trabaja por la infancia en situación de vulnerabilidad, que en 2014 midió las habilidades para la vida de niñas y niños de forma objetiva y científica, enfocando tres áreas del bienestar: las habilidades fundamentales, los activos del desarrollo y el alfabetismo funcional, los resultados obtenidos reflejaron una gran preocupación frente al uso positivo del tiempo libre, donde los adolescentes manifiestan mayores desafíos y limitados espacios de participación en sus distintos contextos familiares, comunitarios, escolares y sociales(21), resultados similares a los encontrados en este estudio, donde los niños y adolescentes referían que la comunidad no los tenía en cuenta en los distintos espacios de participación y por esta razón era más frecuente el desinterés por las problemáticas de la Parroquia, sin embargo, posteriormente se logró propiciar espacios de interacción entre niños, jóvenes y la comunidad dándoles voz y voto en la toma de decisiones.

El fortalecimiento del sentido de comunidad en los niños y adolescentes permitió la identificación y apropiación de tradiciones, símbolos y costumbres que los representan, integrándose a las distintas actividades Parroquiales, lo cual hace referencia a dos conceptos clave que manejó Escuela Móvil: comunidad, como un grupo humano enmarcado en un espacio geográfico determinado que comparte en lo fundamental afinidad de actitudes, sentimientos, tradiciones, usos y patrones lingüísticos correspondientes a una lengua histórica o idioma ${ }^{(22)}$ y territorio, que más allá de sus características económicas, ecológicas y geográficas, se define por los aspectos sociales y culturales que le otorgan singularidad y un significado simbólico(23).

Por otra parte, los resultados encontrados en Escuela Móvil muestran la relación existente entre el desarrollo de habilidades para la vida y la adquisición de características que permite a los niños y jóvenes reconocerse, reconocer al otro, establecer adecuadas relaciones interpersonales y ser asertivos en la toma de decisiones, esto 
concuerda con el estudio realizado por González, et al.(24), quienes proporcionan evidencia de la efectividad del desarrollo de habilidades las cuales permiten la solución de problemas, reconocimiento y expresión de sentimientos y una mejor interacción con los pares y adultos.

Frente al enfoque de derechos humanos trabajados por Escuela Móvil, los resultados mostraron la importancia de su abordaje, los niños y adolescentes fortalecieron valores que les permitieron reconocer al otro y además guiar sus acciones hacia un bien común. Lo anterior tiene relación con lo propuesto por Arias( ${ }^{(25)}$ quien al analizar varios estudios sobre los derechos de niños y jóvenes encontró que a través de estos procesos los participantes fueron cada vez más respetuosos con los derechos de los demás, mostrando cambios positivos de actitud y comportamiento, reflejando la importancia de su enseñanza siendo recursos eficaces para promover la justicia social.

A partir de los ejes que se trabajaron los niños y adolescentes fortalecieron su autoconocimiento, comunicación asertiva, empatía y relaciones interpersonales, siendo habilidades que se incrementan con más frecuencia en la aplicación de estudios similares como el que propone Choque y Chirinos ${ }^{(4)}$, quienes mediante un programa educativo de desarrollo de habilidades para la vida con adolescentes escolarizados obtuvieron como resultado el incremento significativo en habilidades de comunicación y asertividad. De igual manera, el estudio realizado por Morales, et al.(6), quienes implementaron dos programas de intervención psicoeducativa basados en habilidades para la vida destacaron una mejora en habilidades sociales.

Frente a la realidad social y las diversas situaciones cotidianas que se presentan en la Parroquia, el interés del proceso investigativo radicaba en que los niños fortalecieran la toma de decisiones, los participantes aprendieron que antes de dar una opinión o decidir debían tomar un tiempo para pensar, desarrollándose la habilidad de tomar decisiones(26) y de solución de problemas y conflictos, que está relacionada con los procesos de conducta y de pensamiento dirigidos a la ejecución de una tarea intelectualmente exigente que busca una alternativa adecuada como respuesta a un problema, el cual es una oportunidad de cambio, crecimiento personal y social(27).

\section{Conclusiones}

A través de la estrategia de Escuela Móvil se logró el empoderamiento de los niños y adolescentes como sujetos de derechos, los participantes reconocieron claramente sus derechos fundamentales, los deberes por los cuales deben responder y además participan de manera activa y constante de la realidad comunitaria y promueven escenarios que les permitan solventar las necesidades básicas.

La investigación permitió constatar que, a partir de la adquisición, desarrollo y fortalecimiento de habilidades para la vida en niños y adolescentes, se pueden establecer sanas relaciones en diferentes contextos.

Se evidenció la importancia de que la población infantil y adolescente de la Parroquia Quiroga sea partícipe de los distintos procesos comunitarios, permitiendo un reconocimiento de la realidad social, aquellas situaciones cotidianas a las que deben enfrentarse y tengan la capacidad de empoderarse y actuar frente a las mismas, siendo sujetos activos y propositivos.

Se recomienda que, en el desarrollo de estrategias como Escuela Móvil, exista una participación constante de las personas que conforman los contextos cercanos a los niños y adolescentes y puedan contribuir al proceso, además de garantizar la creación de capacidades instaladas que posteriormente sigan adelantando las actividades y no requieran de actores externos a la comunidad.

Conflicto de intereses: Ninguno.

\section{Referencias}

1. Acosta A, Mayoral F. Situación económica y ambiental de Ecuador en un entorno de crisis internacional. Quito: FLACSO; 2013. Available from: http://www.flacsoandes.edu.ec/libros/digital/54216.pdf

2. Ministerio Coordinador de Desarrollo Social. Informe de desarrollo social 2007 - 2017. Quito: Ministerio Coordinador de Desarrollo Social; 2017 [citado 1 Mar 2018]. Disponible en: http://www.desarrollosocial.gob.ec/wpcontent/uploads/2017/03/libro_social-2007-2017.pdf

3. Gómez M. Educación popular, alternativas pedagógicas y sistematización de experiencias. Historia y horizontes. Praxis \& Saber [Internet]. 2015; 6(12):129-148. Available from:

http://www.scielo.org.co/pdf/prasa/v6n12/v6n12a07.pdf 
4. Choque R, Chirinos J. Eficacia del Programa de Habilidades para la Vida en Adolescentes Escolares de Huancavelica, Perú. Rev. Salud Pública [Internet]. 2009; 11(2):169-181. Available from: http://www.scielo.org.co/pdf/rsap/v11n2/v11n1a02.pdf

5. Díaz L, Rosero R, Melo M, Aponte D. Habilidades para la vida: análisis de las propiedades psicométricas de un test creado para su medición. Revista Colombiana de Ciencias Sociales [Internet]. 2013; 4(2):181-200. Available from: https://www.redalyc.org/articulo.oa?id=497856283003

6. Morales M, Benítez M, Agustín D. Habilidades para la vida (cognitivas y sociales) en adolescentes de una zona rural. REDIE [Internet]. 2013; 5(3):98-113. Available from: http://www.redalyc.org/articulo.oa?id=15529662007

7. Martínez V. Habilidades para la vida: una propuesta de formación humana. Itinerario Educativo [Internet]. 2014; (63):61-89. Available from: www.revistas.usb.edu.co/index.php/Itinerario/article/do wnload/1488/1257

8. Magendzo A, Toledo M. Educación en derechos humanos: Estrategia pedagógica-didáctica centrada en la controversia. Educare [Internet]. 2015; 19(3):1-16. Available from: http://www.revistas.una.ac.cr/index.php/EDUCARE/articl e/view/6864/7036

9. Camaño A. Niño sujeto de derechos, en centros educativos privados de primera infancia. [Tesis de pregrado]. Montevideo: Universidad República de Uruguay; 2015

10. Lozano A. Los derechos del niño: cuestiones sobre su fundamentación. Rev. Latinoam cienc soc niñez juv [Internet]. 2016; 14(1):67-79. Available from: http://www.scielo.org.co/pdf/rlcs/v14n1/v14n1a04.pdf

11. Dávila $P$, Naya L. La protección de la infancia en situaciones de riesgo en América Latina a través de los códigos de la niñez. Pedagogía Social. Revista Interuniversitaria [Internet]. 2012; (19):99-112. Available from: http://www.redalyc.org/articulo.oa?id=135025474008

12. Del Canto E. Investigación y métodos cualitativos: un abordaje teórico desde un nuevo paradigma. Ciencias de la educación [Internet]. 2012; 22(40):181-199. Available from:

http://servicio.bc.uc.edu.ve/educacion/revista/n40/art09. pdf

13. Melero N. El paradigma crítico y los aportes de la investigación acción participativa en la transformación de la realidad: un análisis desde las ciencias sociales. Cuestiones pedagógicas [Internet]. 2011; 21,339-355. Available from: https://institucional.us.es/revistas/cuestiones/21/art_14. pdf

14. Oliveira G. Investigación Acción Participativa: una alternativa para la epistemología social en Latinoamérica. Revista de Investigación [Internet]. 2015; 39(86):271-290. Available http://www.scielo.org.ve/pdf/ri/v39n86/art14.pdf

15. Rekalde I, Vizcarra M, Macazaga A. La observación como estrategia de investigación para construir contextos de aprendizaje y fomentar procesos participativos. Educación XX1 [Internet]. 2014; 17(1):201-220. Available from: http://www.redalyc.org/pdf/706/70629509009.pdf
16. Espinoza R, Ríos S. El diario de campo como instrumento para lograr una práctica reflexiva. En: XIV Congreso Nacional de Investigación Educativa, San Luis Potosí: 2017. p. 1-11.

17. Guerrero P, Aguirre C, Besser C, Morales M, Salinas J, Zamora, M. Talleres socioeducativos en infancia desde un enfoque situado. Revista Latinoamericana de Ciencias Sociales, Niñez y Juventud [Internet]. 2017; 15 (1):249-265. Available http://www.scielo.org.co/pdf/rlcs/v15n1/v15n1a16.pdf

18. Código Ético del Psicólogo, Colombia. Revista Latinoamericana de Psicología [Internet]. 2000; 32(1):209225. Available from: http://www.redalyc.org/articulo.oa?id=80532121

19. Martínez M. La Investigación Cualitativa (Síntesis Conceptual). Revista de investigación en psicología [Internet]. 2006; 9(1):123-146. Available from: http://sisbib.unmsm.edu.pe/bvrevistas/investigacion_psic ologia/v09_n1/pdf/a09v9n1.pdf

20. Sequera M. Investigación acción: un método de investigación educativa para la sociedad actual. Revista Arjé (Internet). 2016; 10(18):223-229. Available from: http://arje.bc.uc.edu.ve/arj18/art23.pdf

21. Edex.es [Internet]. Costa Rica: Chahin; 2016 [actualizado 20 Ene 2018; citado 30 Ene 2018]. Disponible en: http://www.edex.es/world-vision-costa-rica-evalua-sutrabajo-en-habilidades-para-la-vida/

22. Liceaga G. El concepto de comunidad en las ciencias sociales latinoamericanas: apuntes para su comprensión. Cuadernos Americanos [Internet]. 2013; 145(3):57-85. Available from: http://www.cialc.unam.mx/cuadamer/textos/ca14557.pdf

23. Spíndola 0. Espacio, territorio y territorialidad: una aproximación teórica a la frontera. Revista Mexicana de Ciencias Políticas y Sociales [Internet]. 2016; 61(228):2755. Available from: https://www.redalyc.org/articulo.oa?id=42149082003

24. González C, Ampudia A, Guevara Y. Programa de intervención para el desarrollo de habilidades sociales en niños institucionalizados. Acta Colombiana de Psicología [Internet]. 2012; 15 (2):43-52. Available from: http://www.scielo.org.co/pdf/acp/v15n2/v15n2a05.pdf

25. Arias B. La infancia como sujeto de derechos. Un análisis crítico. Ratio Juris [Internet]. 2017; 12(24):127-142. Available from: https://dialnet.unirioja.es/servlet/articulo?codigo=67489 76

26. Luna A, Laca F. Patrones de toma de decisiones y autoconfianza en adolescentes bachilleres. Revista de Psicología [Internet]. 2014; 32(1):39-65. Disponible en: http://www.redalyc.org/pdf/3378/337831261002.pdf

27. Garaigordobil M, Maganto C. Empatía y resolución de conflictos durante la infancia y la adolescencia. Rev Latinoam psicol [Internet]. 2011; 43(2):255-266. Available from:

http://www.scielo.org.co/pdf/rlps/v43n2/v43n2a05.pdf 